\title{
Preparing PAMAM-NK4 nano complexes and examining their in vitro growth suppression effects in breast cancer
}

\author{
Minfeng Liu ${ }^{1 \#}$, Zhaoze Guo $^{1 \#}$, Jiangqin Liu ${ }^{1 \#}$, Hui Ren ${ }^{2}$, Jingyun Guo ${ }^{1}$, Shijun Liao ${ }^{1}$, Zicheng Zhang ${ }^{1}$ \\ ${ }^{1}$ Breast Center, Department of General Surgery, Nanfang Hospital, Southern Medical University, Guangzhou, China; ${ }^{2}$ Transfusion Medicine, School \\ of Laboratory Medicine and Biotechnology, Southern Medical University, Guangzhou, China \\ Contributions: (I) Conception and design: M Liu; (II) Administrative support: M Liu; (III) Provision of study materials or patients: Z Guo; (IV) \\ Collection and assembly of data: J Guo; (V) Data analysis and interpretation: Z Guo, J Liu, J Guo; (VI) Manuscript writing: All authors; (VII) Final \\ approval of manuscript: All authors. \\ \#These authors contributed equally to this work. \\ Correspondence to: Minfeng Liu. Breast Center, Department of General Surgery, Nanfang Hospital, Southern Medical University, Guangzhou 510515, \\ China. Email: matthew1@smu.edu.cn.
}

Background: This study sought to examine the suppression of the NK4 (which is a fragment that originates from the trypsin digestion of the hepatocyte growth factor) gene as mediated by new nano material polyamidoamine (PAMAM) dendrimers in the growth of breast cancer cells MDAMB-231 and MCF-7, and the therapeutic effects in a nude mice model of transplanted tumor cell MDA-MB-231.

Methods: We built PAMAM-NK4 nano particles and detected the in vitro transfection rate. Nano complexes and blank plasmid PAMAM dendrimers were transfected to MDA-MB-231 and MCF-7 cells, respectively. The western-blotting method, MTT experiment method, and bead method were used to detect the effects of the nano complexes on NK4 protein expression, cell proliferation, and cell apoptosis. The nude mice model of transplanted tumor cell MDA-MB-231 comprised 40 nude female mice who were subject to injections. The mice were randomly divided into four groups, comprising 10 mice per group. The control, blank plasmid and treatment groups were subcutaneously injected with $0.2 \mathrm{~mL}$ of $0.9 \% \mathrm{NaCl}$ (Sodium chloride) solution, $0.2 \mathrm{~mL}$ of plasmid solution (including $100 \mu \mathrm{g}$ PAMAM pcDNA3.1(-) blank plasmid nano complexes) and $0.2 \mathrm{~mL}$ of plasmid solution (including PAMAM-NK4 $100 \mu \mathrm{g}$ ) beside the tumor inoculation spot, respectively. The positive control group was intraperitoneally injected with $0.2 \mathrm{~mL}$ of doxorubicin solution, including $100 \mu \mathrm{g}$ doxorubicin. Western blotting was used to detect the NK4 protein expression of the transplanted tumor tissues of the various groups.

Results: NK4 protein was successfully expressed in MDA-MB-231 and MCF-7 cells transfected with PAMAM-NK4 nano particles, and cell proliferation was suppressed and cell apoptosis was induced. The tumor volumes and masses of the treatment and positive control groups were obviously smaller than those of the control group. The differences were statistically significant $(\mathrm{P}<0.05)$. The treatment group had an obviously higher mean value of $N K 4$ protein expression than the control group. The differences were statistically significant $(\mathrm{P}<0.05)$.

Conclusions: PAMAM-NK4 nano complexes suppress the growth of the breast cancer cells MDA-MB-231 and MCF-7, and had a treatment effect on this tumor nude mice model of breast cancer cells.

Keywords: Polyamidoamine (PAMAM); NK4; breast cancer; genetic treatment

Submitted May 20, 2021. Accepted for publication Sep 17, 2021.

doi: $10.21037 /$ gs-21-443

View this article at: https://dx.doi.org/10.21037/gs-21-443 


\section{Introduction}

Breast cancer is presently one of the most common female malignant tumors $(1,2)$. It severely affects the physical and psychological health of women all over the world. We have been striving to find a safer yet more efficient treatment method for breast cancer. Further study in the areas of genomics and molecular biology, and knowledge of the advantages of genetic treatments have led to wider application, lower toxicities, better targeting and more stable expression in tumor treatment (3). Currently, many related genes are used in the study of breast cancer $(4,5)$. However, the key gene occurrence mechanism of breast cancer remains unknown. Thus, a more effective target gene needs to be identified.

$N K 4$ is a fragment that originates from the trypsin digestion of the hepatocyte growth factor $(H G F)(6,7)$. It is composed of 477 amino acids and 4 kringle areas at the $\mathrm{N}$ end of the $\alpha$ chain of the $H G F$ molecule. When the $H G F$ and its receptor, c-Met, combine, biological effects are produced. The key part of the compound is located between the hairpin-shaped structure and kringle area at the $\mathrm{N}$ end of the $\alpha$ chain. By this crucial structure, NK4 is able to totally and competitively suppress the interaction between $H G F$ and c-Met without inducing a phosphorylation of the c-Met, which contributes to the obstruction of the signal transduction pathway of $H G F / c-M e t$ and suppresses the tumor cell growth, invasion, and metastasis induced by $H G F$ (8-10). NK4 also prevents the generation of blood vessels. Previous research has shown that that NK4 suppresses the generation of new capillaries in gallbladder, lung and liver cancer tumors (10-13). This experiment used the NK4 gene as a target gene to study the suppression effects of the NK4 gene in breast cancer.

An appropriate carrier is of great importance for the purpose of efficient gene transfection and stable gene expression. The lipid carrier (14) is safe, and has low transfection efficiency; virus (15) has a high transfection rate, however, its target gene capacity is low, and its immunogenicity and toxicity are strong. Polyamidoamine (PAMAM) dendrimers (16-18) can be used to solve this problem. PAMAM dendrimers have the following advantages: they have a relatively high transfection efficiency; they prevent deoxyribonucleic acid (DNA) from being destroyed; and they extend the preservation term of DNA. Additionally, experiments have shown that PAMAM dendrimers can transfect target genes, such as the curcumin target gene, to breast cancer cells $(19,20)$. PAMAM dendrimers also have transfection features of low toxicity and high efficiency.

This study combined PAMAM dendrimers (as carriers) with the target gene, NK4. We prepared the PAMAM-NK4 nano complexes and observed role of its in suppressing breast in in vitro and in vivo experiments to provide a theoretical basis for the clinical study of genetic treatments for breast cancer. We present the following article in accordance with the ARRIVE reporting checklist (available at https://dx.doi.org/10.21037/gs-21-443).

\section{Methods}

\section{Major materials}

The human breast cancer cell lines MDA-MB-231 and MCF-7 were purchased from the Shanghai Institute of Cell Biology. BALB/c-nu/nu mice (female, aged 4 to 6-week-old, and weighing 14-19 g) were purchased from experimental animal centers of the Southern Medical University (Experimental Animal Certificate No.: SCXK-Y 2011-0015). All animal experiments were performed under a project license (No. NFYY-2021-131) granted by institutional ethics committee of the Nanfang Hospital, are in compliance with the relevant regulations of the China Animal Protection Committee for the care and use of animals. Recombinant plasmid pcDNA3.1NK4 and blank plasmid pcDNA3.1(-), which was used in the control group, were preserved by Institute of Tumor of Southern Medical University. The 5th generation of PAMAM nano material, trypsinase, Dulbecco's Modified Eagle Medium (DMEM), and dimethyl sulfoxide (DMSO) were purchased from the American company, Sigma. The primary antibody, marked with NK4 (human HGF mAb), and the secondary rabbit anti-mouse antibody, marked with horseradish peroxidase, were purchased from the American company Leinco. Cell lysate was purchased from Shanghai Beyotime Biological Technology Co., Ltd. Fetal bovine serum (FBS) was purchased from the American company, Gibco. Doxorubicin was purchased from Shenzhen Main Luck Pharmaceutical Inc., batch number 0209E2. ECL (Electrochemical luminescence) test kits were purchased from the American company, Thermo Pierce. BCA protein quantitative analysis test kits were purchased from Shanghai Yubo Biologycal Co., Ltd. AnnexinV-FITC apoptosis test kits and propidium iodide (PI) were purchased from Nanjing KeyGEN BioTECH. 


\section{Preparing the PAMAM-NK4 nano complexes}

PAMAM and recombinant plasmid pcDNA3.1-NK4 were combined at a charge ratio of $5: 1$, and vibrated and mixed for $15 \mathrm{~s}$. After being mixed, they were kept at an ambient temperature for $30 \mathrm{~min}$. PAMAM and blank plasmid pcDNA3.1(-) were combined using the same method to prepare the blank plasmid nano complexes.

\section{Transfecting MCF-7 and MDA-MB-231 cell lines of buman breast cancer}

MDA-MB-231 and MCF-7 cells were inoculated in a 6-hole medium at a density of $1 \times 10^{5} /$ hole. The incubated cell density was about $50-70 \%$. The cells were washed with PBS twice. The blank plasmid nano complexes and the PAMAM-NK4 nano complexes were diluted with the same amount of non-serum DMEM into the suspension of final concentration of $100 \mathrm{nmol} / \mathrm{L}$. Each suspension with $2 \mathrm{~mL}$ was taken for cell transfection and cultured in a $5 \%$-carbon dioxide $\left(\mathrm{CO}_{2}\right)$ incubator at $37{ }^{\circ} \mathrm{C}$. The culture medium was changed to a DMEM containing $10 \%$ FBS 4 h later, and left to incubate for another $24 \mathrm{~h}$. The experiment was repeated 3 times.

\section{Detecting the in vitro transfection rates}

The transfected cells prepared using the above method were incubated for another $24 \mathrm{~h}$, and then observed with a fluorescence microscope to detect green fluorescent cells to confirm that the cells had been positively transfected. Five vision fields were randomly selected and the amount of positively transfected cells and the total number of cells in each vision field were counted. The transfection rate was calculated using the following formula: Transfection rate = number of positively transfected cells/total number of cells $\times 100 \%$.

\section{Examining the effects of PAMAM-NK4 transfection on the NK4 protein expression of breast cancer cells using the western-blot detection method}

Cells in the exponential phase were taken from the control, blank plasmid, and treatment groups. A cell protein sample was obtained using cell lysate, and the BCA method was used to measure the total protein concentration of each sample. NK4 primary antibody (human $H G F$ mAb) was added to the samples, at $4{ }^{\circ} \mathrm{C}$, and stored overnight, after the total protein stripes of the samples had been electrophoretically separated, transferred to film and closed by sodium dodecyl sulfate polyacrylamide gel electrophoresis. Tris buffered saline was used to wash the films 3 times, and a secondary antibody with a HRP marker was added with an expression of $\beta$-actin. The films were washed and then developed using ECL chemical luminescence test kits. The films were scanned, and IPP6.0 software was used to analyze the gray value of the stripes. The experiment was repeated 3 times.

\section{Examining the effects of PAMAM-NK4 transfection on the proliferation of breast cancer cells using the MMT detection method}

MDA-MB-231 and MCF-7 cell lines in the exponential phase were inoculated in 96-hole plates at a density of $5 \times 10^{4} /$ hole and divided into a control group (not transfected), a blank plasmid group [transfected with the blank plasmid nano complex PAMAM- pcDNA3.1(-)], and a treatment group (transfected with the PAMAM-NK4 nano complex). There were 5 duel-holes in each group with $40 \mu \mathrm{L}$ MTT solution, which was replaced 4 h later with clean solution. $150 \mu \mathrm{L}$ of DMSO was added and vibrated for 10 min. Blank holes (culture medium + DMSO) were also provided. The blank holes were set as 0 with an optical density (OD) value of $570 \mathrm{~nm}$ for each hole and were measured using an enzyme-linked immunosorbent assay detector to compare differences in the proliferation abilities of the cells among the groups. The following formula was use: Proliferation suppression rate $(\%)=(1-$ OD value of treatment group/OD value of control group) $\times 100 \%$.

\section{Examining the effects of PAMAM-NK4 transfection on the apoptosis of breast cancer cells using the bead method}

Cell suspension was taken from the control, blank plasmid, and treatment group, and inoculated in 6-hole plates at a density of $1 \times 10^{5} /$ hole and cultured in a $5 \%$ $\mathrm{CO}_{2}$ incubator at $37^{\circ} \mathrm{C}$ for $3 \mathrm{~h}$, after which the culture medium was discarded. Cells were collected after being washed with phosphate-buffered saline (PBS) twice and were cultured with appropriate amounts of AnnexinVfluorescein isothiocyanate (FITC) and PI, and kept at room temperature for $15 \mathrm{~min}$ away from light. A beam analyzer was used to analyze the cell apoptosis rates. 
Establishing a transplanted tumor nude mice model of buman breast cancer cells

MDA-MB-231 cells in the exponential phase were taken and processed into cell suspension of $5 \times 10^{7} / \mathrm{mL}$ with a non-serum medium after trypsin digestion. Suspension with $0.2 \mathrm{~mL}$ (containing $1 \times 10^{7} \mathrm{MDA}-\mathrm{MB}-231$ cells) was subcutaneously injected into the nude mice at the fat pad of the second nipple on the left. The 40 nude mice were injected. Thereafter, observations and measurements were taken every 2 days to check the growth of the tumor nodules, and daily after the 7 th day. The long diameter (I) and short diameter (II) were measured using a vernier caliper and recorded to calculate tumor volume (V). The following formula was used: $\mathrm{V}=\mathrm{ab}^{2} / 2$.

\section{Detecting the effects of PAMAM-NK4 on tumor growth in the tumor-bearing mice model}

The tumor-bearing mice were randomly divided into four groups, comprising 10 mice each. Mice in the control group were subcutaneously injected with $0.2 \mathrm{~mL}$ of $0.9 \%$ $\mathrm{NaCl}$ solution beside the tumor inoculation spot. Mice in the blank plasmid group were subcutaneously injected with $0.2 \mathrm{~mL}$ plasmid solution, including $100 \mu \mathrm{g}$ PAMAMpcDNA3.1(-) blank plasmid nano complexes, beside the tumor inoculation spot. Mice in the treatment group were subcutaneously injected with $0.2 \mathrm{~mL}$ of plasmid solution, including PAMAM-NK4 $100 \mu \mathrm{g}$, beside the tumor inoculation spot. Mice in the positive control group were intraperitoneally injected with $0.2 \mathrm{~mL}$ doxorubicin solution, including $100 \mu \mathrm{g}$ doxorubicin. Detection began on the 7 th day of cell injection, and the injections were administered daily until the 14th day. The survival status of the tumor-bearing mice was observed daily and the mortality rate of each group was recorded. The long and short diameters of the tumors were measured every other day. Specifically, the longest diameter (I) was measured first, followed by the longest axes vertical to the longest diameter (IIb). The following calculation formula was used: tumor volume $(\mathrm{V})=\mathrm{ab}^{2} / 2$. The tumor-bearing mice were killed on the 30th day and the tumors were completely removed. Adhesion to surrounding tissue and skin was observed. PBS was used to wash the blood on the tumors. Tumor mass was taken by an electronic balance when the tumors had dried.

\section{Examining the effects of PAMAM-NK4 on the NK4 protein expression of the transplanted tumor tissues using the western-blot detection method}

Transplanted tumor tissue in $0.2 \mathrm{~g}$ was taken from each group, and then cut and ground in lysate. Supernatant was sucked up after the centrifugal procedure to measure cell protein concentration. The western-blot method (see section 1.5) was used to detect the NK4 protein expression of the transplanted tumor tissue of the various groups.

\section{Statistic analysis}

The data were analyzed using SPSS 19.0 statistical software, and measurement materials were presented in the form of mean value \pm standard deviation $(\bar{x} \pm s)$. To ensure that no variance among the groups was missed, the Welch method was adopted to perform inter-group comparisons, and Dunnett's T3 method was used for the pair comparison; otherwise, a one-way analysis of variance was conducted to perform the inter-group comparisons, and the least significant differences method was used for the pair comparisons. A $\mathrm{P}<0.05$ was considered statistically significant.

\section{Results}

\section{Detecting the in vitro transfection rates}

Green fluorescent cells were found in the breast cancer cell lines $24 \mathrm{~h}$ after they were transfected with PAMAMNK4 nano complexes. Green fluorescent protein was found in both the MDA-MB-231 and MCF-7 cells. Fluorescent expression increased as time went by and reached its peak at $48 \mathrm{~h}$, which was when transfection efficiency was at its highest. The MDA-MB-231 cells had more green fluorescent protein expression than the other types of cells (see Figure 1). Fluorescence began to weaken after $72 \mathrm{~h}$.

\section{Examining the effects of PAMAM-NK4 nano complex transfection on the NK4 protein expression of human breast cancer cells using the western-blot detection method}

$H G F$ expression (of the endogenous NK4 protein) was found in the MDA-MB-231 and MCF-7 cells in the control group, each of which had mean values of $0.88 \pm 0.03$ and 
$0.35 \pm 0.01$, respectively. The $N K 4$ protein expression of the MDA-MB-231 cells was higher than that of the MCF7 cells (see Figure 2). The mean value of NK4 protein expression of the MDA-MB-231 and MCF-7 cells in the blank plasmid group after transfection were $0.87 \pm 0.03$ and $0.37 \pm 0.01$, respectively. The difference between the blank plasmid and control groups was not statistically significant $(\mathrm{P}>0.05)$.

The mean values of the NK4 protein expression of the
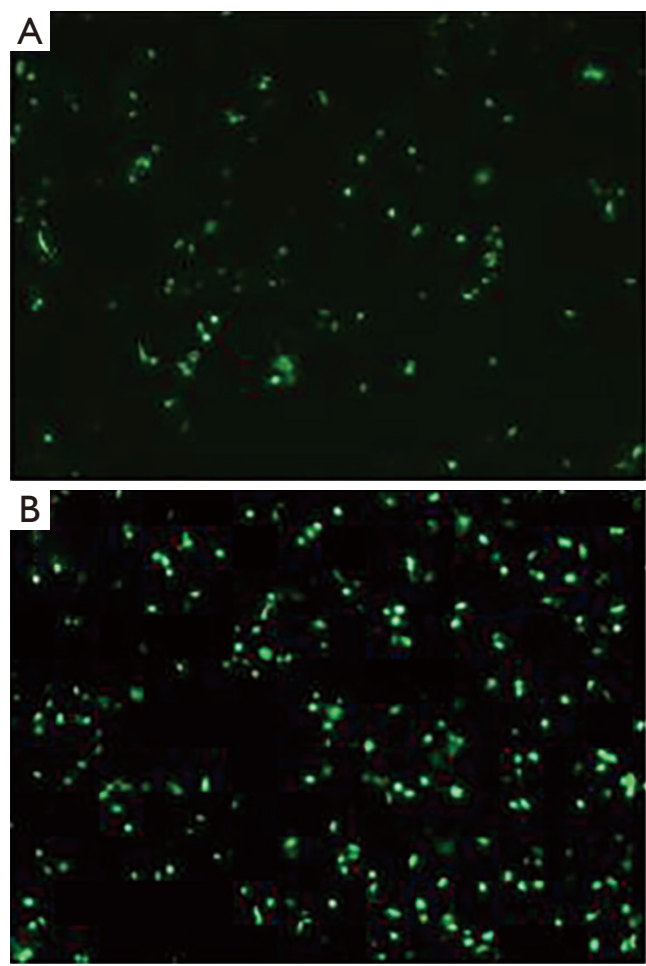

Figure 1 The results of a cellular transfection test as visualized by a fluorescence microscope. (A) MCF-7; (B) MDA-MB-231. Magnification: (A) 40×; (B) 100×.
MDA-MB-231 and MCF-7 cells in the treatment group were $2.26 \pm 0.02$ and $1.26 \pm 0.01$, respectively. Compared to the control group, the NK4 protein expression of the two cell lines obviously increased after being transfected with PAMAM-NK4 nano complexes; the differences were statistically significant $(\mathrm{P}<0.05)$. Additionally, the NK4 protein expression of the MDA-MB-231 cells was higher than that of the MCF-7 cells.

\section{Examining the effects of PAMAM-NK4 nano complex transfection on the proliferation ability of human breast cancer cells using the MTT method}

There was no significant difference between the proliferation ability of the cells in the blank plasmid and control groups (see Figure 3). Compared to the control group, the OD values of the MDA-MB-231 $(0.27 \pm 0.06 v s$. $0.54 \pm 0.06)$ and MCF-7 $(0.30 \pm 0.05$ vs. $0.43 \pm 0.05)$ cells in the treatment group were obviously decreased; the differences were statistically significant (both $\mathrm{P}<0.05$ ). In relation to the two cell lines, the suppression rate of the MDA-MB-231 cells was $50.4 \%$, which was obviously higher than that of the MCF-7 cells (30.6\%); the difference was statistically significant $(\mathrm{P}=0.01)$.

\section{Examining the effects of PAMAM-NK4 nano complex transfection on the apoptosis rate of breast cancer cells using the bead method}

The percentages of the apoptosis rates of the MDAMB-231 and MCF-7 cells in the blank plasmid group were $2.21 \% \pm 0.04 \%$ and $2.39 \% \pm 0.03 \%$, respectively; however, the difference between these groups and the control group was not statistically significant. In relation to the comparison between the treatment and control groups, the percentage differences of the cell apoptosis rates were as follows: MDA-

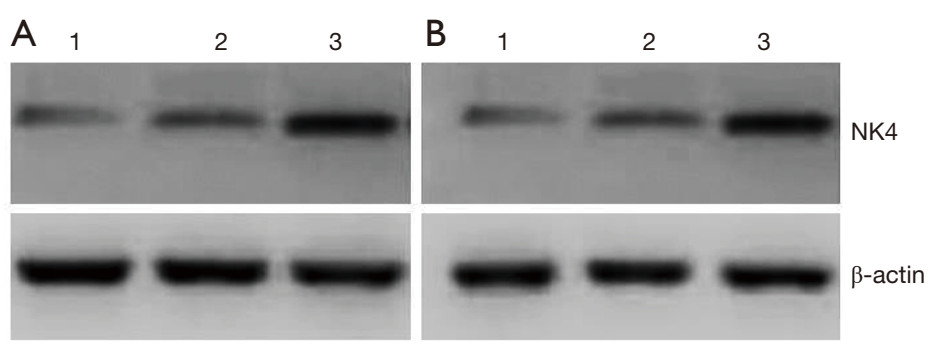

Figure 2 Expressions of NK4 protein in human breast cancer cells increased after PAMAM-NK4 transfection. (A) MCF-7; (B) MDAMB-231. 1: Control; 2: Blank plasmid; 3: PAMAM-NK4. 
MB-231 cells: $4.21 \% \pm 0.03 \%$ vs. $2.26 \% \pm 0.06 \%$; and MCF7 cells: $3.95 \% \pm 0.05 \%$ vs. $2.40 \% \pm 0.04 \%$. The apoptosis rate of the treatment group was obviously higher than that of the control group; the difference was statistically significant. The apoptosis rate of the MDA-MB-231 cells was obviously higher than that of the MCF-7 cells (see Figure 4).

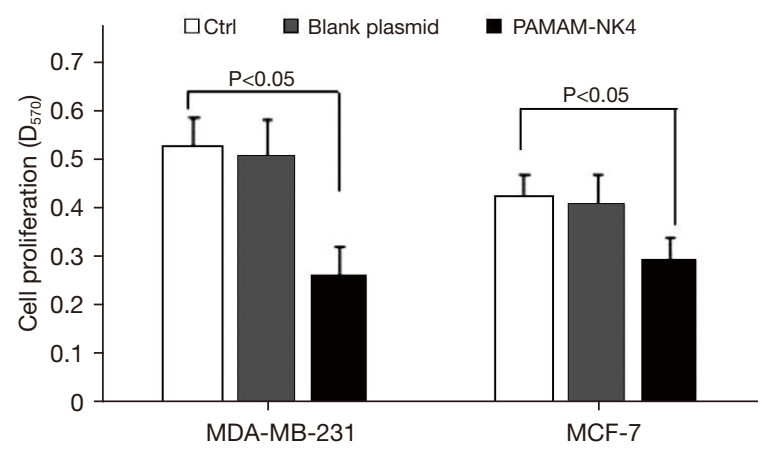

Figure 3 The proliferation of human breast cancer cells was inhibited by PAMAM-NK4 transfection.

\section{Successfully establishing a nude mice cell transplanted model of buman breast cancer}

Subcutaneous nodules were observed at inoculating spots $1 \mathrm{w}$ after inoculation. The masses grew slowly in round or elliptical shapes. No swelling or ulceration was found at the inoculation spots (see Figure 5). The tumor occurrence rate was $100 \%$. The general condition of the tumor-bearing mice in the groups was satisfactory. The mice displayed no obvious change in appetite, sleeping patterns, urine or stools, spirit or activity.

\section{Examining the effects of PAMAM-NK4 nano complex transfection on the NK4 protein expression of transplanted tumor cell using the western-blot detection method}

The mean value of the NK4 protein expression of the control group was $0.82 \pm 0.05$. The mean values of the NK4 protein expression of the blank plasmid and positive control groups were $0.87 \pm 0.11$ and $0.84 \pm 0.08$, respectively; there were no significant differences between these groups
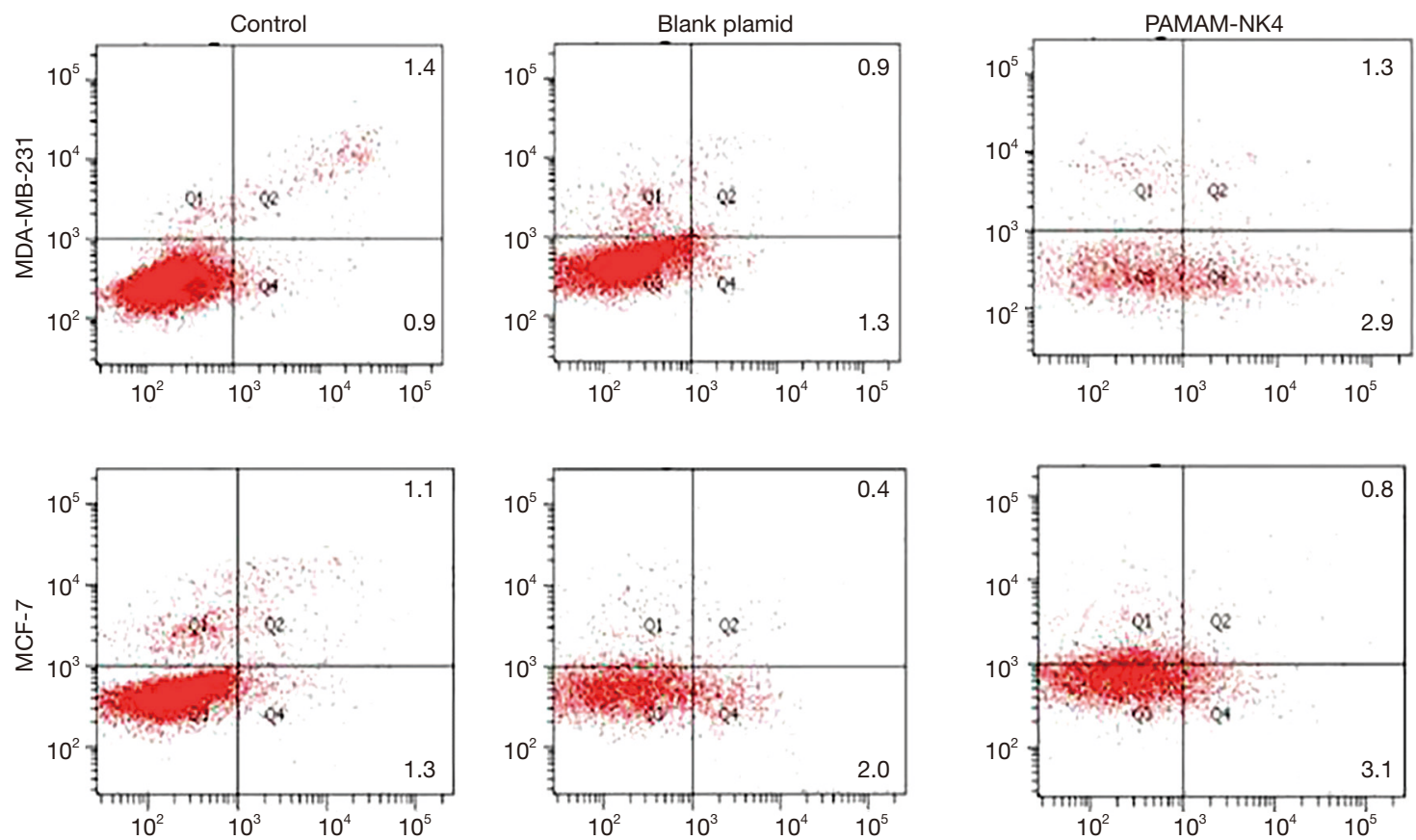

Figure 4 Apoptosis rates of breast cancer cells MDA-MB-231 and MCF-7 (enhanced by the overexpression of NK4). 


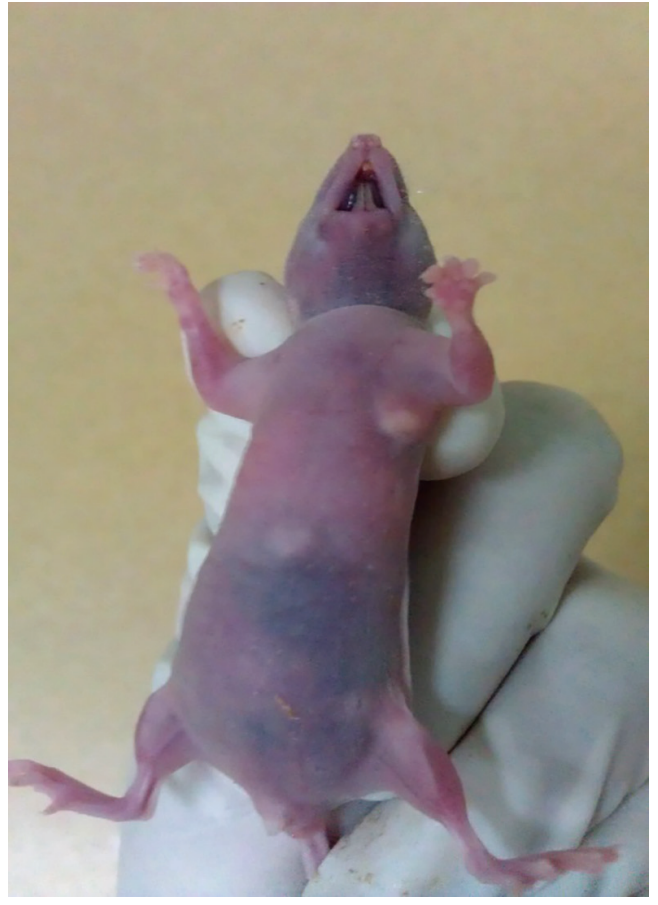

Figure 5 Nude mice transfected with the human breast cancer model.

A

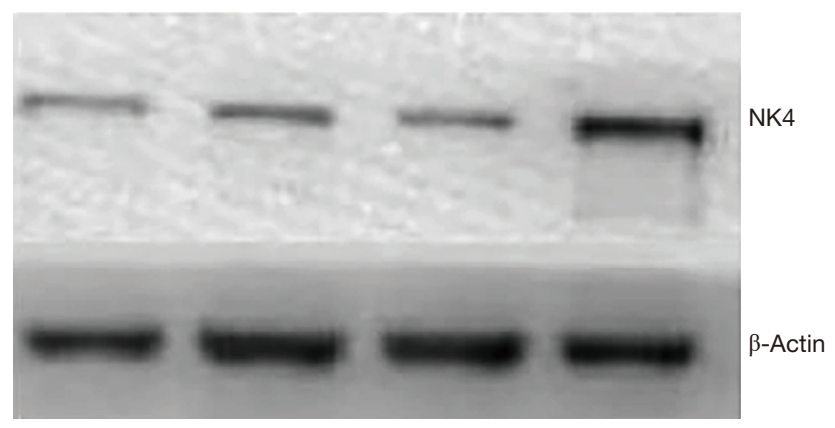

Figure 6 Expressions of $N K 4$ protein in the transplanted tumor cells of various groups detected using a western-blot assay. (A) Control; (B) blank plasmid; (C) positive control; (D) PAMAMNK4.

and the control group $(\mathrm{P}>0.05)$. The mean value of the NK4 protein expression of the treatment group, which was transfected with the PAMAM-NK4 nano complex, was $3.44 \pm 0.07$ (see Figure 6). Compared to that of the control group, the protein expression amount of the treatment group was high, and the difference was statistically significant.

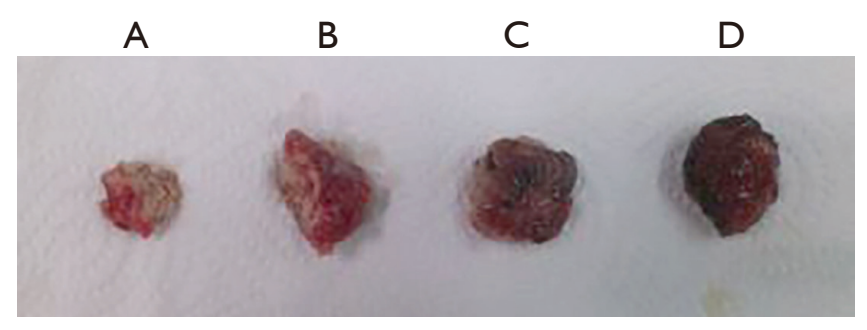

Figure 7 Tumor tissues of nude mice from various groups. (A) PAMAM-NK4; (B) positive control; (C) blank plasmid; (D) control.

\section{PAMAM-NK4 suppressed the growth of transplanted tumors in tumor-bearing mice}

The administration of cell suspension was continuously performed, and survival status and tumor diameter were monitored. No tumor-bearing mice died from an adverse reaction during the period of culture. Tumor-bearing mice were killed on the 30th day, and the transplanted tumors were completely removed (see Figure 7). The tumors were elliptical masses, observable by the naked eye. Most had a complete capsule. A few tumors adhered to surrounding tissues or skin and had a tenacious texture.

Tumor diameter was measured to calculate the volume. Tumor mass was measured by an electronic balance. As Figure 8 shows, the tumor volume of the control group was $2.42 \pm 0.62 \mathrm{~cm}^{3}$, that of the blank plasmid group was $2.38 \pm 0.64 \mathrm{~cm}^{3}$, that of the treatment group was $1.50_{ \pm}$ $0.43 \mathrm{~cm}^{3}$, that of the positive control group was $1.64 \pm$ $0.31 \mathrm{~cm}^{3}$, and that of the control group was $3.86 \pm 1.12 \mathrm{~g}$. The tumor mass of the blank plasmid group was $3.48 \pm$ $1.07 \mathrm{~g}$, that of the treatment group was $1.41 \pm 0.47 \mathrm{~g}$, and that of the positive control group was $1.54 \pm 0.38 \mathrm{~g}$. There was no significant difference in tumor volume and mass between the control and blank plasmid groups $(\mathrm{P}>0.05)$; the tumor volume and mass of the treatment and positive control groups were obviously smaller than those of the control group. The differences were statistically significant. There was no significant differences between the treatment and positive control groups $(\mathrm{P}>0.05)$.

\section{Discussion}

After extensive research on the causes of breast cancer, genetic treatment is considered one of the best potential options for the comprehensive treatment of breast cancer. To perform a treatment successfully, the appropriate target genes must be identified and an effective gene carrier must 

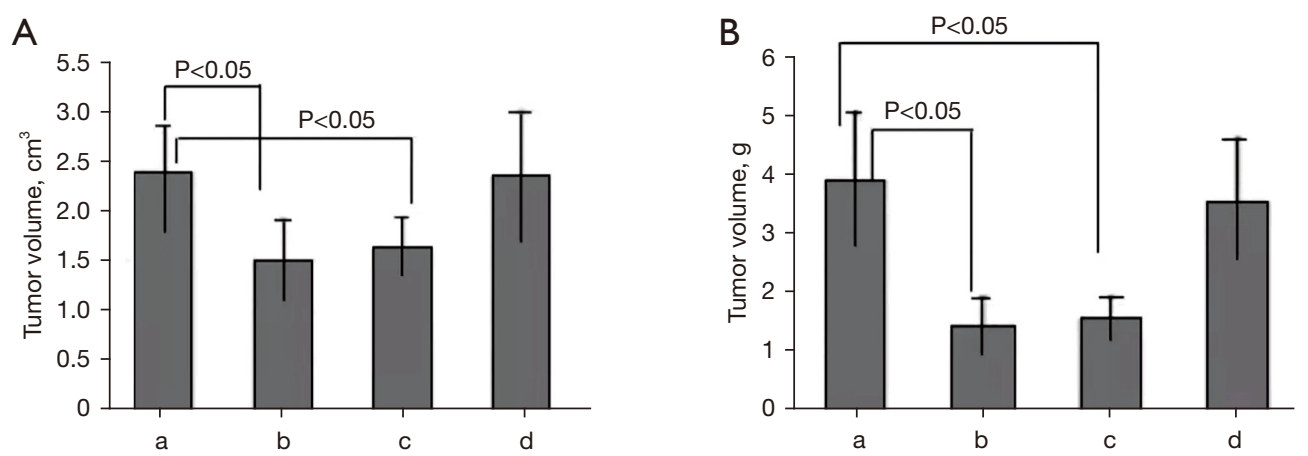

Figure 8 Tumor volumes (A), mass (B) and tumor blocks of nude mice xenografts in various groups on day 30. a: control; b: PAMAM-NK4; c: positive control; d: blank plasmid.

transfect the target gene into the target cells. The NK4 gene was selected as the target gene in this experiment. Research has shown that transfection efficiency is at its highest when PAMAM and DNA are combined at a charge ratio of 5:1 (21). Thus, in this experiment, PAMAM-NK4 nano complexes were prepared according to guidelines to transfect different breast cancer cell lines (i.e., MDAMB-231 and MCF-7). In relation to the transfection, green fluorescent protein was found in both the MDAMB-231 and MCF-7 cells, which showed that PAMAM had successfully introduced the recombinant plasmid pcDNA3.1-NK4 into the two breast cancer cell lines. The expression of green fluorescent protein was stronger in MDA-MB-231 cells than MCF-7 cells, which means that the transfection efficiency of the nano complex was higher in the MDA-MB-231 cells. A western-blot analysis revealed endogenous $H G F$ expression in both the breast cancer in control groups, and the cell content of MDA-MB-231 was slightly higher than that of MCF-7 cells. The MDAMB-231 cell is a Basal-like-type breast cancer cell line, while the MCF-7 cell is a Luminal A-type breast cancer cell line (22). In relation to the molecular branch, the malignancy of the MDA-MB-231 cell was higher; thus, $H G F$ content may be related to the malignancy of the breast cancer. The expression of NK4 protein was significantly increased in the treatment group compared to the control group, which implies the higher expression of the exogenous NK4 protein.

There were no significant statistical differences in the cell proliferation and apoptosis rates between the blank plasmid (PAMAM) and control groups. Thus, PAMAM did not affect cell proliferation and apoptosis and is biologically safe (without outstanding cytotoxicity). In both the treatment and control groups, the two types of cells showed decreased proliferation abilities and increased apoptosis rates. Thus, the proliferation of both breast cancer cells, which were transfected with PAMAM-NK4 nano complexes, was greatly suppressed, which resulted in higher apoptosis rates. The extent of proliferation suppression and the apoptosis rates of the MDA-MB-231 cells were relatively higher than those of MCF-7 cells. Thus, the suppression effects of the nano complexes were more obvious in the MDA-MB-231 cells than the MCF-7 cells.

To determine if the nano complexes had the same anticancer effects in animals, the method of original injection (with cell suspension) was applied to a prepare tumorbearing mice transplanted tumor model. The results of the comparison showed that the MDA-MB-231 cells had a higher transfection rate of nano complexes and a stronger suppression effect against tumors than the MCF-7 cells. Thus, the MDA-MB-231 cell line was selected to establish the human breast cancer transplanted tumor nude mice model.

Subcutaneous masses were observed at the injection spot $1 \mathrm{w}$ after the inoculation was performed. The tumor occurrence was $100 \%$, and the general conditions of the mice were favorable. After administration, no obvious changes were observed in the appetite, sleeping pattern, urine and stools, spirit, and activity of the tumor-bearing mice, and no tumor-bearing mice died from an adverse reaction. These results demonstrate PAMAM's safety; no obvious adverse in vivo reactions were observed. The NK4 protein was expressed in the transplanted tumors of the mice in the treatment group, according to the western-blot results, which is evidence of the PAMAM carrier's successful in vivo transfection of a target gene to a tumor cell and stable expression.

The tumor masses and volumes of the treatment and 
positive control groups obviously declined compared to those of the control group. Thus, the nano complexes and traditional chemical treatment medicine doxorubicin effectively suppressed the growth of tumors. The PAMAMNK4 nano complexes stably expressed NK4 protein in vitro and in vivo, and significantly suppressed the growth of breast cancer cells, which contributed to the anti-tumor effects.

Notably, no significant statistical differences were found between the treatment and positive control groups, which indicates that while genetic treatments had the same anticancer effects as chemical treatments, genetic treatments do not outweigh chemical treatments as the best treatment method. Genetic treatments can be used to treat breast cancer; however, challenges remain. This study showed that much shall be fulfilled in the way of genetic treatments and indicates directions for future research.

\section{Acknowledgments}

Funding: The Basics and Applied Basic Research Foundation of Guangdong Province of China (Grant No. 2021A1515011651).

\section{Footnote}

Reporting Checklist: The authors have completed the ARRIVE reporting checklist. Available at https://dx.doi. org/10.21037/gs-21-443

Data Sharing Statement: Available at https://dx.doi. org/10.21037/gs-21-443

Conflicts of Interest: All authors have completed the ICMJE uniform disclosure form (available at https://dx.doi. org/10.21037/gs-21-443). The authors have no conflicts of interest to declare.

Ethical Statement: The authors are accountable for all aspects of the work in ensuring that questions related to the accuracy or integrity of any part of the work are appropriately investigated and resolved. All animal experiments were performed under a project license (No. NFYY-2021-131) granted by institutional ethics committee of the Nanfang Hospital, are in compliance with the relevant regulations of the China Animal Protection Committee for the care and use of animals.
Open Access Statement: This is an Open Access article distributed in accordance with the Creative Commons Attribution-NonCommercial-NoDerivs 4.0 International License (CC BY-NC-ND 4.0), which permits the noncommercial replication and distribution of the article with the strict proviso that no changes or edits are made and the original work is properly cited (including links to both the formal publication through the relevant DOI and the license). See: https://creativecommons.org/licenses/by-nc-nd/4.0/.

\section{References}

1. Torre LA, Bray F, Siegel RL, et al. Global cancer statistics, 2012. CA Cancer J Clin 2015;65:87-108.

2. Renzulli M, Zanotti S, Clemente A, et al. Hereditary breast cancer: screening and risk reducing surgery. Gland Surg 2019;8:S142-9.

3. Naik R, Veldore VH, Gopinath KS. Genetics and Breast Cancer - Oncologists Perspectives. Indian J Surg Oncol 2015;6:415-9.

4. Wang T, Zhang J, Xiao J, et al. Mutational analysis of BRCA1 and BRCA2 in northwest Chinese breast cancer patients. Transl Cancer Res 2019;8:1845-52.

5. Holstege H, Joosse SA, van Oostrom CT, et al. High incidence of protein-truncating TP53 mutations in BRCA1-related breast cancer. Cancer Res 2009;69:3625-33.

6. Date K, Matsumoto K, Shimura H, et al. HGF/NK4 is a specific antagonist for pleiotrophic actions of hepatocyte growth factor. FEBS Lett 1997;420:1-6.

7. Date K, Matsumoto K, Kuba K, et al. Inhibition of tumor growth and invasion by a four-kringle antagonist (HGF/NK4) for hepatocyte growth factor. Oncogene 1998;17:3045-54.

8. Garajová I, Giovannetti E, Biasco G, et al. c-Met as a Target for Personalized Therapy. Transl Oncogenomics 2015;7:13-31

9. Zhang J, Jiang X, Jiang Y, et al. Recent advances in the development of dual VEGFR and c-Met small molecule inhibitors as anticancer drugs. Eur J Med Chem 2016;108:495-504.

10. Mizuno S, Nakamura T. HGF-MET cascade, a key target for inhibiting cancer metastasis: the impact of NK4 discovery on cancer biology and therapeutics. Int J Mol Sci 2013;14:888-919.

11. Parr C, Jiang WG. Hepatocyte growth factor activators, inhibitors and antagonists and their implication in cancer 
intervention. Histol Histopathol 2001;16:251-68.

12. Boissinot M, Vilaine M, Hermouet S. The Hepatocyte Growth Factor (HGF)/Met Axis: A Neglected Target in the Treatment of Chronic Myeloproliferative Neoplasms? Cancers (Basel) 2014;6:1631-69.

13. Rabquer BJ, Koch AE. NK4 therapy: a new approach to target angiogenesis and inflammation in rheumatoid arthritis. Arthritis Res Ther 2013;15:119.

14. Felgner JH, Kumar R, Sridhar CN, et al. Enhanced gene delivery and mechanism studies with a novel series of cationic lipid formulations. J Biol Chem 1994;269:25 50-61.

15. Otsuka M, Baru M, Delrivière L, et al. In vivo liverdirected gene transfer in rats and pigs with large anionic multilamellar liposomes: routes of administration and effects of surgical manipulations on transfection efficiency. J Drug Target 2000;8:267-79.

16. Wang $\mathrm{M}, \mathrm{Hu} \mathrm{H}$, Sun $\mathrm{Y}$, et al. A pH-sensitive gene delivery system based on folic acid-PEG-chitosan - PAMAMplasmid DNA complexes for cancer cell targeting. Biomaterials 2013;34:10120-32.

17. Daneshvar N, Abdullah R, Shamsabadi FT, et al. PAMAM dendrimer roles in gene delivery methods and stem cell

Cite this article as: Liu M, Guo Z, Liu J, Ren H, Guo J, Liao S, Zhang Z. Preparing PAMAM-NK4 nano complexes and examining their in vitro growth suppression effects in breast cancer. Gland Surg 2021;10(9):2695-2704. doi: 10.21037/gs$21-443$ research. Cell Biol Int 2013;37:415-9.

18. Jin L, Zeng X, Liu M, et al. Current progress in gene delivery technology based on chemical methods and nanocarriers. Theranostics 2014;4:240-55.

19. Atapour MH, Mojarad M, Raoofian R, et al. PAMAM Megamer (G2-G2) as a versatile tool in gene delivery. Clinical Biochemistry 2011;44:S281-2.

20. Mollazade M, Zarghami N, Nasiri M, et al. Polyamidoamine (PAMAM) encapsulated curcumin inhibits telomerase activity in breast cancer cell line. Clinical Biochemistry 2011;44:S217.

21. Malik N, Wiwattanapatapee R, Klopsch R, et al. Dendrimers: relationship between structure and biocompatibility in vitro, and preliminary studies on the biodistribution of 125I-labelled polyamidoamine dendrimers in vivo. J Control Release 2000;65:133-48.

22. Subik K, Lee JF, Baxter L, et al. The Expression Patterns of ER, PR, HER2, CK5/6, EGFR, Ki-67 and AR by Immunohistochemical Analysis in Breast Cancer Cell Lines. Breast Cancer (Auckl) 2010;4:35-41.

(English Language Editor: L. Huleatt) 